\title{
Numerical Analysis and Comparison of Gridless Partial Differential Equations
}

\author{
Zhao Zhang, \\ Public Teaching Department, Zhumadian Vocational and Technical College, \\ Zhumadian, 463000 \\ China
}

Received: February 22, 2021. Revised: August 9, 2021. Accepted: August 28, 2021. Published: August 31, 2021.

\begin{abstract}
In the field of science and engineering, partial differential equations play an important role in the process of transforming physical phenomena into mathematical models. Therefore, it is very important to get a numerical solution with high accuracy. In solving linear partial differential equations, meshless solution is a very important method. Based on this, we propose the numerical solution analysis and comparison of meshless partial differential equations (PDEs). It is found that the interaction between the numerical solutions of gridless PDEs is better, and the absolute error and relative error are lower, which proves the superiority of the numerical solutions of gridless PDEs.
\end{abstract}

Keywords-Background grid integration, meshless, numerical solution, partial differential equation.

\section{INTRODUCTION}

$\mathrm{W}$ ITH the development of science and technology and society, a large number of complex computing problems appear in front of people. Before the advent of computers, in order to solve some complex computing problems, many scientists devoted most of their lives, even their whole lives. In 1867, French astronomer Dalamny spent 20 years to solve a perturbation series of celestial motion Expansion [1]. But this is not a good way to solve complex problems, so people began to study the methods to solve complex calculation problems. In order to solve some complex calculation problems, numerical calculation methods appear. The numerical solution of PDEs is one of the most important branches. For example, to accurately predict the weather change, thousands of PDEs must be solved [2]. It is unrealistic to solve them manually. Therefore, the numerical solution of PDEs is very important. There are three kinds of numerical methods for PDEs, the finite difference method, the variation method and the finite element method. The most common one is the finite difference method.
However, the finite difference method will be unstable when it is used to solve the partial differential equation. Therefore, it is necessary to analyze the stability of the finite difference method in solving the partial differential equation. The stability of the difference equation refers to the study of the influence of the initial value interference on the solution of the difference equation when there is no error in the right free term of the difference equation. It reflects the situation that whether the difference solution is continuous depends on the initial value there are many kinds of difference schemes.

The essence of numerical method is discretization and algebra: Discretization transforms an infinite information system into a finite information system, and algebra transforms a partial differential equation into a computer solvable algebraic equation. No matter what kind of numerical method, it involves the division of spatial discrete element. The practice shows that the quality of discrete element directly affects the accuracy and precision of calculation results. The commonly used spatial discrete element is grid, and there are two kinds of grid currently used: Structural grid and unstructured grid generation technology. Although the current generation technology of structural grid and unstructured grid has been quite mature, but there are some inherent advantages and disadvantages, especially with the problem to be solved more and more complex, the importance of spatial discretization method is more and more prominent. Therefore, the main difference between the mesh free method and the finite element method is the function approximation. In the finite element method, piecewise polynomial interpolation is mainly used for function approximation. This method needs to mesh the solution area in advance, and the pre-processing is complicated. The meshless method overcomes this disadvantage, and does not need to divide the grid, only needs the node information. There are many function approximation methods without grid. For example, radial basis function interpolation method, moving least square method, approximate method, integral kernel approximate method and unit decomposition method, etc. 


\section{Numerical SOlution ANALysis Of Meshless PARTIAL DIFFERENTIAL EQUATION}

\section{A. Gridless and Slightly Scattered Data Interpolation}

A group of scattered data in gridless PDEs, For example, at point $\mathrm{x}_{\mathrm{i}} \in R^{\mathrm{s}}(\mathrm{i}=1, \ldots, N)$, We got the data $\mathrm{y}_{\mathrm{i}}$, We need to fit a smooth function $\operatorname{Pf}(\mathrm{x})$ through this set of scattered data sampling points, in order to obtain the function value of any position, the function strives to spread the information related to scattered points smoothly to all positions in the definition domain. This problem is of great practical value in many fields of science and engineering, because the data that can be measured or generated in practice are often sparse and irregularly distributed [3]. They can be found in various scientific and engineering applications, such as geology, meteorology, oceanography, surveying and mapping, and mining, where they often collect some non-uniform measurement results of physical quantities; Experimental data of scattered distribution in chemistry, physics and engineering; Calculation results of non-uniform distribution of output when solving partial differential equation with finite element method, etc. The goal of scattered data interpolation is to reconstruct a basic function to meet the given set of data. At each point, there are:

$$
\operatorname{Pf}\left(\mathrm{x}_{\mathrm{i}}\right)=\mathrm{y}_{\mathrm{i}}
$$

This method is usually called interpolation method. A common and convenient method to solve scattered data interpolation that is the assumption $\operatorname{Pf}\left(\mathrm{x}_{\mathrm{i}}\right)$ is some linear combination of $B_{\mathrm{k}}(\mathrm{x})$, that is, interpolation in a linear space:

$$
\operatorname{Pf}(\mathrm{x})=\sum_{\mathrm{i}=1}^{N} \mathrm{c}_{\mathrm{i}} B_{\mathrm{i}}(\mathrm{x})
$$

In this way, the original interpolation problem is transformed into a solution system of equations:

$$
A \mathrm{c}=\mathrm{y}
$$

Among them:

$$
\begin{aligned}
A & =\left[\begin{array}{cccc}
B_{1}\left(\mathrm{x}_{1}\right) & B_{2}\left(\mathrm{x}_{1}\right) & \cdots & B_{\mathrm{N}}\left(\mathrm{x}_{1}\right) \\
B_{2}\left(\mathrm{x}_{2}\right) & B_{2}\left(\mathrm{x}_{2}\right) & \cdots & B_{\mathrm{N}}\left(\mathrm{x}_{2}\right) \\
\vdots & \vdots & \ddots & \vdots \\
B_{1}\left(\mathrm{x}_{\mathrm{N}}\right) & B_{2}\left(\mathrm{x}_{\mathrm{N}}\right) & \cdots & B_{\mathrm{N}}\left(\mathrm{x}_{\mathrm{N}}\right)
\end{array}\right] \\
\mathrm{c} & =\left(\mathrm{c}_{1}, \mathrm{c}_{2}, \ldots \mathrm{c}_{N}\right)^{\mathrm{t}}, \mathrm{y}=\left(\mathrm{y}_{1}, \mathrm{y}_{2}, \ldots, \mathrm{y}_{N}\right)^{\mathrm{t}}
\end{aligned}
$$

Obviously, under this assumption, the original problem has a unique solution if and only if matrix A is nonsingular.

This problem is understood in one-dimensional case $(\mathrm{s}=1)$, the commonly used polynomial interpolation uses the polynomial of degree $N-1$ to interpolate at any $\mathrm{N}$ different points [4]. It is easy to know that there is always a unique solution. But for multidimensional situations $(\mathrm{s} \geq 2)$, this conclusion is not necessarily true. For example, in the two-dimensional case, when these $\mathrm{n}$ points are in the unit circle: $\mathrm{x}^{2}+\mathrm{y}^{2}=1$, Then any interpolation function plus $\mathrm{x}^{2}+\mathrm{y}^{2}-1$ of any times still satisfies the interpolation condition, that is, it cannot satisfy the uniqueness.

For the convenience of studying this problem, in a real valued continuous function, the positive definite function on RS means that it is even function, and for any $\mathrm{n}$ different points $\mathrm{x}_{1}, \ldots, \mathrm{x}_{N} \in R^{\mathrm{s}}, \mathrm{c}=\left(\mathrm{c}_{1}, \mathrm{c}_{2}, \ldots, \mathrm{c}_{\mathrm{N}}\right)^{\mathrm{t}} \in R^{N}$ there are:

$\sum_{\mathrm{i}=1}^{N} \sum_{\mathrm{j}=1}^{N} \mathrm{c}_{\mathrm{i}} \mathrm{c}_{\mathrm{j}} \Phi\left(\mathrm{x}_{\mathrm{i}}-\mathrm{x}_{\mathrm{j}}\right) \geq 0$

Note: $\Phi \in P D_{\mathrm{s}}$ (positive define). Function is equal sign in strict positive formula (6), it's only true if $\mathrm{C}$ is zero.

It can be seen from the definition of strictly positive definite function, if $\Phi$ is strictly positive definite, then the matrix $A=\left(\begin{array}{cccc}\Phi\left(\mathrm{x}_{1}-\mathrm{x}_{1}\right) & \Phi\left(\mathrm{x}_{2}-\mathrm{x}_{1}\right) & \cdots & \Phi\left(\mathrm{x}_{N}-\mathrm{x}_{1}\right) \\ \Phi\left(\mathrm{x}_{1}-\mathrm{x}_{2}\right) & \Phi\left(\mathrm{x}_{2}-\mathrm{x}_{2}\right) & \cdots & \Phi\left(\mathrm{x}_{N}-\mathrm{x}_{2}\right) \\ \vdots & \vdots & \ddots & \vdots \\ \Phi\left(\mathrm{x}_{1}-\mathrm{x}_{N}\right) & \Phi\left(\mathrm{x}_{2}-\mathrm{x}_{N}\right) & \cdots & \Phi\left(\mathrm{x}_{N}-\mathrm{x}_{N}\right)\end{array}\right)$ is positive and certainly reversible.

Through the calculation, the mesh free partial micro scattered random data interpolation is obtained, and the preliminary positioning of the equation value is achieved.

\section{B. Numerical Setting Function}

According to the above calculation, if the function is strictly positive definite, if function $\Phi$ is a strictly positive definite function, if interpolation base function $\mathrm{B}_{\mathrm{k}}(\mathrm{X})$ is taken as $\Phi\left(\mathrm{x}-\mathrm{x}_{\mathrm{k}}\right)$, then there must be a unique solution [5]. As for how to judge whether a function $\Phi$ is a positive definite function, the following theory proposed by Bochne can be used:

The Fourier transform of Function $\mathrm{f} \in L_{1}\left(R^{\mathrm{s}}\right)$ is defined as:

$F \mathrm{f}(\mathrm{w})=\int_{R^{\mathrm{s}}} \mathrm{f}(\mathrm{x}) \mathrm{e}^{-2} \mathrm{dx}$

The inverse Fourier transform of:

$F^{-1} \mathrm{f}(\mathrm{x})=\int_{R^{s}} \mathrm{f}(\mathrm{w}) \mathrm{e}^{-2} \mathrm{dw}$

Function $\Phi \in C\left(R^{\mathrm{s}}\right)$ is a positive definite function on $R^{\mathrm{s}}$ if and only if it is a Fourier transform called by a finite nonnegative Borel measure on $R^{\mathrm{s}}$, that is:

$\Phi(\mathrm{x})=\mu(\mathrm{x})=\int_{R^{s}} \mathrm{e}-\mathrm{d} \mu(\mathrm{y})$

In order to make matrix A positive definite, function $\Phi$ must be strictly positive definite, Bochner's theorem only gives the condition of positive definite function $\Phi$, for this reason, we must extend Bochner's theorem and give the condition of strictly positive definite in function [6]:

Suppose $\mu$ is a finite nonnegative Borel measure on $R^{\mathrm{s}}$, and its support set is not a Lebesgue zero measure set. Then the Fourier transform called is a strictly positive definite function on $R^{\mathrm{s}}$.

Inference gives a method to construct strictly positive definite function

Suppose function $f \in L_{1}\left(R^{\mathrm{s}}\right)$ is a nonzero nonnegative continuous function, then the Fourier transform of $\mathrm{F}$ is a strictly positive definite function on $R^{\mathrm{s}}$. 
The specific method to determine whether a given function is a strictly positive definite function can be given by the following theorem:

Suppose $\Phi \in L_{1}\left(R^{\mathrm{s}}\right)$ is a continuous function. $\Phi$ is a positive definite function if and only if $\Phi$ is bounded and the Fourier transform of $\Phi$ is nonnegative and not equal to zero function.

$\Phi=\mathrm{e}^{-\alpha\|x\| 2}$ is $R^{\mathrm{s}}$ strictly positive definite function on (for any s). This is because the Fourier transform of Gaussian function or Gaussian function, in particular, when $\alpha=\frac{1}{2}$, $F \Phi=\Phi$, this can be proved directly by calculation.

\section{Positive Definite Radial Function}

A multivariable function $\Phi: R^{\mathrm{s}} \rightarrow R$ called radial function, it means that there is a single variable function $\Phi:[0,+\infty] \rightarrow R$.

Radial function has many advantages, such as keeping the function value unchanged after rotation and reflection. The most important thing is that using radial function as the basis function interpolation has little to do with the dimension s of space [7]. We only need to use a one variable function to do various operations instead of a multi variable function. When the dimension of space becomes larger, the complexity will increase dramatically.

A univariate function $\Phi$ is the (strict) positive definite radial function on RS, which means that its corresponding multivariable function $\Phi$ is the (strict) positive definite function on $R^{\mathrm{s}}$ and is the radial function. Note that when function $\Phi$ is determined, its positive definiteness is generally determined by the dimension s of space $R^{\mathrm{s}}$.

The method of judging whether a given function must be a positive definite radial function on $R^{\mathrm{s}}$ can be given by Schoenberg's theorem:

Continuous function $\Phi:[0,+\infty] \rightarrow R$ is a positive definite radial function on $R^{\mathrm{s}}$ if and only if it is a Bessel transformation of a finite nonnegative Borel measure $\mu$ on $[0,+\infty]$, that is:

$$
\Phi(\mathrm{r})=\int_{0}^{\infty} \Omega(\mathrm{rt}) \mathrm{d} \mu(\mathrm{t})
$$

Among them:

$\Omega_{\mathrm{s}}(\mathrm{rt})= \begin{cases}\cos _{\mathrm{r}} & \mathrm{s}=1 \\ \left(\frac{\mathrm{s}}{2}\right)\left(\frac{2}{\mathrm{r}}\right)^{(\mathrm{s}-2) / 2} & s \geq 2\end{cases}$

Among them: $\mathrm{r}=\|\mathrm{x}\|$ is the norm of $\mathrm{x} \in R^{\mathrm{s}}$.

If function $\Phi$ is a (strictly) positive definite radial function on $R^{\mathrm{s}}$, that is to say, its corresponding multivariable function satisfies any $\mathrm{n}$ different points $\mathrm{x}_{1}, \ldots, \mathrm{x}_{N} \in R^{\mathrm{s}}, \mathrm{c}=\left(\mathrm{c}_{1}, \mathrm{c}_{2}, \ldots, \mathrm{c}_{N}\right)^{\mathrm{t}} \in R^{N} \quad$ [8]. That is, the function must also be a (strictly) positive definite radial function on $R^{\mathrm{s}}$. Another function $\Phi:(0,+\infty) \rightarrow R$ is the positive definite radial function on all $R^{\mathrm{s}}$ (for all $\mathrm{s}$ ), that is, for all $R^{\mathrm{s}}$, the function $\Phi(\mathrm{x})=\Phi(\|\mathrm{x}\|)\left(\mathrm{x} \in R^{\mathrm{s}}\right)$ on $R^{\mathrm{s}}$ is the positive definite radial function.

Continuous function $\Phi:(0,+\infty) \rightarrow R$ is a positive definite radial function on all $R^{\mathrm{s}}$ if and only if it can be expressed in the following form:

$$
\Phi(\mathrm{r})=\int_{0}^{\infty} \mathrm{e}^{-\mathrm{r} 2 \mathrm{t} 2} \mathrm{~d} \mu(\mathrm{t})
$$

where $\mu$ is a finite nonnegative Borel measure over $(0,+\infty)$, thus a positive definite radial function is obtained.

\section{Weighted Allocation of Basis Function}

Least square method considering weighting:

$\int_{\Omega}\left(P(D)\left(\sum \lambda_{j} \phi_{j}(x)\right)-f(x)\right)^{2} w_{1}(x) d x$

$\left.+\int_{\partial \Omega}\left(Q(D) \sum \lambda_{j} \phi_{j}(x)\right)-g(x)\right)^{2} w_{2}(x) d x$

If you select $w_{1}(x)=\sum_{j=1}^{n} \delta(x-x j)(x j \in \Omega)$, the configuration method is exported. The collocation method uses the basis function to approximate the solution as follows:

$u(x)=\sum_{j} \lambda_{j} \phi_{j}(x)$

Then select dense point $\{x j\}_{j=1}^{n} \in \Omega \quad\{x k\}_{k=n+1}^{n+m} \in \partial \Omega$ and force equations and boundary conditions to hold on to these selected points:

$P(D) u\left(x_{j}\right)=f\left(x_{j}\right), j=1, \ldots, n$

$Q(D) u\left(x_{k}\right)=g\left(x_{k}\right), k=n+1, \ldots, n+m$

There are two problems: One is the unique solvability of the discrete equation; The other is whether the solution of the discrete equation converges to the real when the data points are dense in the discussion area [9]. According to the positive definite radial function formula above, can make $L_{j} u=P(D) u\left(x_{j}\right), L_{k} u=Q(D) u\left(x_{k}\right)$, in this way, the discrete partial differential equation becomes a Hermite-Birkhof interpolation problem. If the boundary value problem of partial differential equation is well posed, then the system of linear equations of Hermite-Birkhof interpolation is the only solvable one. If so: If $\varphi(w)<(1+|w|)^{-2 l-d}$, the order of the PDE under consideration is $\mathrm{m}$, so when the boundary value problem of PDE is stable, the error between the solution of the discrete equation and the real solution of the equation can be estimated as follows: $\left\|u^{*(\alpha)}(x)-u^{(\alpha)}(x)\right\| \leq(h)^{l-m-|\alpha|}$

Here $h$ is the density of the data points. In this way, we get a method of numerical solution of PDE, which only needs to arrange points without triangulation. The basic function of collocation method is $\phi_{j}(x)=L_{j y} \phi(\|x-y\|)$, therefore:

$$
\begin{aligned}
& P(D) \sum_{j} \lambda_{j} L_{j y} \phi(\|y-x\|)=\left.f(x)\right|_{x=x_{k}}, k=1, \ldots, n \\
& Q(D) \sum_{j} \lambda_{j} L_{j y} \phi(\|y-x\|)=\left.g(x)\right|_{x=x_{k}}, k=n+1, \ldots, n+m
\end{aligned}
$$




\section{E. Numerical Solution of Two Dimensional Laplace Equation Without Grid}

After the above numerical calculation, FDM is applied to time, which is transformed into a series of ODE, and then the discrete scheme of ODE is given with FIM, thus the numerical solution is solved iteratively [10].

If the time interval is set to be $\mathrm{t}_{\mathrm{j}}=\mathrm{j}_{\tau}, \mathrm{j}=0,1, \ldots, N$, $\tau=T / N$ and $\mu_{\mathrm{j}}(\mathrm{x})=\mu\left(\mathrm{x}, \mathrm{t}_{\mathrm{j}}\right)$ is recorded, then the implicit eider format is:

$$
\frac{\mu^{\mathrm{j}+1}(\mathrm{x})-\mu^{\mathrm{j}}(\mathrm{x})}{\tau}=\mu_{\mathrm{x}}^{\mathrm{j}+1}(\mathrm{x})+\mathrm{f}\left(\mu^{\mathrm{j}+1}(\mathrm{x})\right)
$$

Among them, $\mathrm{f}(\mu)=-\mu \mu_{\mathrm{x}}$.

This system of nonlinear equations can be treated by implicit explicit method, so as to have the following implicit explicit eider scheme (Euler IMEX):

$-\tau \mu_{\mathrm{x}}^{\mathrm{j}+1}(\mathrm{x})+\mu^{\mathrm{j}+1}(\mathrm{x})=\mu^{\mathrm{j}}(\mathrm{x})+\tau \mathrm{f}\left(\mu^{\mathrm{j}}\right)$

If the center difference scheme is used to discretize the space, the accuracy of the scheme can be proved to be $\mathrm{O}\left(\tau+\mathrm{f}^{2}\right)$.

The implicit and explicit format corresponding to crank Nicolson format can be obtained as follows:

$$
-\tau \mu_{\mathrm{x}}^{\mathrm{j}+1}(\mathrm{x})+2 \mu^{\mathrm{j}+1}(\mathrm{x})=\tau \mu_{\mathrm{x}}^{\mathrm{j}}(\mathrm{x})+2 \tau \mathrm{f}\left(\mu^{\mathrm{j}}\right)
$$

After implicit and explicit processing, the accuracy of this format will be reduced to $\mathrm{O}\left(\tau+\mathrm{f}^{2}\right)$ [11]. Therefore, we can correct it to obtain crank Nicolson's cn-pc:

$$
\left\{\begin{array}{l}
-\tau \mu_{\mathrm{x}}^{\mathrm{j}+1}(\mathrm{x})+2 \mu^{\mathrm{j}+1}(\mathrm{x})=\tau \mu_{\mathrm{x}}^{\mathrm{j}}(\mathrm{x})+2 \mu^{\mathrm{j}}(\mathrm{x})+2 \tau \mathrm{f}\left(\mu^{\mathrm{j}}\right) \\
-\tau \mu_{\mathrm{x}}^{\mathrm{j}+1}(\mathrm{x})+2 \mu^{\mathrm{j}+1}(\mathrm{x})=\tau \mu_{\mathrm{x}}^{\mathrm{j}}(\mathrm{x})+2 \mu^{\mathrm{j}}(\mathrm{x})+\tau \mathrm{f}\left(\mu^{\mathrm{j}}\right)+\tau \mathrm{f}\left(\mu^{\mathrm{j}+1}\right)
\end{array}\right.
$$

where $\mu^{\mathrm{j}+1}$ is the estimated value and $\mu^{\mathrm{j}+1}(\mathrm{x})$ is the corrected value. For the convenience of processing, the known boundary value is also regarded as the unknown function value, so the matrix form of FIM Euler IMEX is given as follows:

$$
\left[\begin{array}{ccc}
\tau \mu-(f)^{2} & \mathrm{x} & 0 \\
f_{1} & 0 & 0 \\
f_{2} & 0 & 0
\end{array}\right]\left[\begin{array}{c}
\mu^{j+1} \\
0 \\
0
\end{array}\right]=\left[\begin{array}{c}
-\left(\mathrm{f}^{(1)}\right)^{2} \mu^{\mathrm{j}}+\frac{1}{2} \mathrm{f}^{(1)} \tau\left(\mu^{\mathrm{j}}\right) 2 \\
\mu\left(0, \tau^{\mathrm{j}+1}\right) \\
\mu\left(1, \tau^{\mathrm{j}+1}\right)
\end{array}\right]
$$

Among them, $f_{1}=[1,0, \ldots, 0], \mathrm{e}_{2}=[0, \ldots, 0,1]$.

In order to further study the implementation mechanism of the meshless method and further understand the implementation details of the method in the numerical solution of partial differential equations, the following two-dimensional Laplace control equations are considered to be solved:

$$
\begin{aligned}
& \frac{\mu^{2} \psi}{\mu \mathrm{x}^{2}}+\frac{\mu^{2} \psi}{\mu \mathrm{y}^{2}}=0 \\
& \left.\psi\right|_{\mathrm{x} 2+\mathrm{y} 2=1}=0
\end{aligned}
$$

where $\psi$ is the flow function, and the equations represent the typical two-dimensional incompressible flow around a cylinder. The following calculation steps are used:

(1) According to the relative position of spatial discrete points, the point cloud is determined. In order to analyze the relationship between point cloud density and calculation accuracy, regular structural grid points are used [12].

(2) The initial value of the inner field and the inner and outer boundary value are given. In order to verify the method and eliminate the influence of boundary conditions on the solution, the analytical solution is used as the outer boundary value

(3) According to the idea of meshless method, the first-order spatial derivatives $\frac{\mu \psi}{\mu \mathrm{x}}$ and $\frac{\mu \psi}{\mu \mathrm{y}}$ of each point in the internal field are fitted with the eigenvalues of points, and then the second-order derivatives $\frac{\mu^{2} \psi}{\mu \mathrm{x}^{2}}$ and $\frac{\mu^{2} \psi}{\mu \mathrm{y}^{2}}$ are fitted with the first-order spatial derivatives.

From this, we can get the large-scale matrix equation group $\mathrm{f}(\psi)=0$, and then calculate the specific numerical value of gridless PDEs.

\section{SimULATION}

In the current computational fluid dynamics (CFD) software design, most of them adopt the structured programming method, and have achieved considerable success. In many years of research and practice, a set of relatively stable design ideas and relatively complete engineering function library have been formed. Most of these softwares are based on the grid to calculate the flow field. The grid cells are closely related with each other and have a large amount of information exchange. Using structured programming language, they can directly access the physical values of grid points and form functional modules with strict structure and high degree of aggregation. However, there are some defects in the structured Liuzi design method: single design goal, insufficient flexibility in the design process, poor scalability, low level of design reuse [13]. According to the object-oriented software design method, it has gradually become a main method of software development. It centers on the objects in the objective world, and its analysis and design ideas are in line with people's thinking mode. The analysis and design results are close to the reality of the objective world.

\section{A. Experimental Preparation}

In order to verify the effectiveness of the numerical analysis and comparison method for meshless partial differential equations proposed in this paper, simulation experiments were carried out. The specific experimental environment is shown in Table I.

Table. I. Experimental environment

\begin{tabular}{ccc}
\hline $\begin{array}{c}\text { Experimental } \\
\text { environment }\end{array}$ & Configuration & Parameter \\
\hline \multirow{2}{*}{ Hardware } & CPU & Intel(R)Core(TM)i5-9400 \\
environment & Frequency & $2.90 \mathrm{GHz}$ \\
& RAM & $16.0 \mathrm{~GB}$ \\
Software & Operating system & Windows 10 \\
environment & Version & 18362.1082 pro \\
& Digits & $64 \mathrm{bit}$ \\
\hline
\end{tabular}




\begin{tabular}{cc}
\hline $\begin{array}{c}\text { Analog software } \\
\text { language } \\
\text { Simulation } \\
\text { software }\end{array}$ & APDL \\
\hline
\end{tabular}

The laboratory environment is shown in Fig. 1.

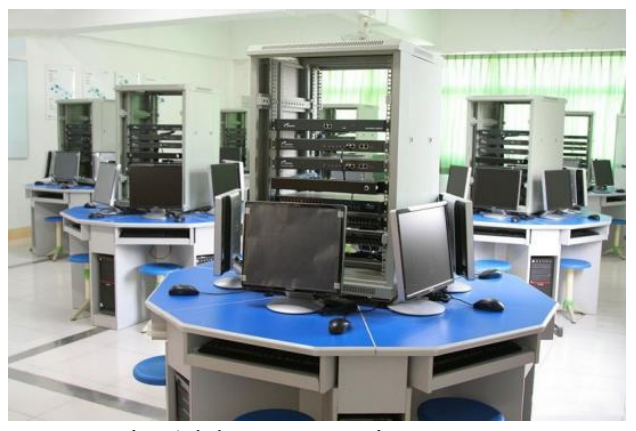

Fig. 1 laboratory environment

The statistical results of experimental data are shown in Table II.

Table. II. Statistical results of experimental data

\begin{tabular}{ccccc}
\hline $\begin{array}{c}\text { Experimental } \\
\text { data volume }\end{array}$ & $\begin{array}{c}\text { Number of } \\
\text { object sets }\end{array}$ & $\begin{array}{c}\text { Number of } \\
\text { state sets }\end{array}$ & $\begin{array}{c}\text { Number of } \\
\text { method } \\
\text { sets }\end{array}$ & $\chi^{2}$ \\
\hline $35 \mathrm{~GB}$ & 1000 & 1000 & 1000 & 0.635 \\
\hline
\end{tabular}

During the experiment, object type is the basic concept of object-oriented technology, which consists of the following parts: object type $=$ object set + state set + operation set + method set + constraint. The object set is a dynamic set of existing instance objects of object type, which changes dynamically with the creation, revocation, entry and exit of objects. An object also has a permanent identifier that satisfies uniqueness, which is called an object identifier. State set is a set of legal states that a class of objects may have. Generally, object set is a static concept, and object state is composed of several state variables (i.e. attributes). The validity of state is determined by the constraint of object type. An operation set is a set of operations that can act on all sets of objects. A method set is a collection of its own operations that an object can call in response to a message. Constraints specify the legal state of this type of object and the timing relationship between the legal states. The instance object is dynamic, and a series of state transitions (i.e. property value changes) can be made through method calls. The object type has a hierarchical structure. The child type automatically constrains the methods, state variables and constraints of the parent type. It also allows the child type to add new methods and state variables, redefine or cancel the parent type methods, but does not allow the weakening of the object determination method.

Design pattern is a description of classes and objects communicating with each other that are used to solve general design problems in specific scenarios. A design pattern names, abstracts and determines the main aspects of a general design structure, which can be used to construct reusable object-oriented design [14]. The design pattern determines the classes and instances involved, their roles, ways of collaboration, and assignment of responsibilities. Brain Foote divides the life cycle of object-oriented software into prototype stage, extension stage and consolidation stage. When software enters the extension stage, its evolution is determined by the following two conflicting requirements:

(a) Meet more needs;

(b) Easy to reuse.

For example, we need software to be able to replace the turbulence model, dynamically generate discrete points according to the actual situation, use different algorithms to generate point cloud structure, etc., and these changes should use the original design as much as possible. Such continuous expansion will often lead to reconstruction. The reconstruction work includes dividing the class into special and general components, and lifting or dropping each operation to the appropriate class at the class level and rationalize the interface of each class. To meet more requirements and reach the requirements of $\mathrm{u}$ higher reusability, the two phases of object-oriented software continue to be expanded and consolidated. One is to expand to meet the new requirements and consolidate to make the software more general. This cycle is inevitable, but good design is robust to the change of requirements, which can avoid refactoring to some extent. Design pattern records the design structure produced by refactoring, and provides the goal for refactoring.

For example, we need software to be able to replace the turbulence model, dynamically generate discrete points according to the actual situation, use different algorithms to generate point cloud structure, etc., and these changes should use the original design as much as possible. Such continuous expansion will often lead to reconstruction. The reconstruction work includes dividing the class into special and general components, and lifting or dropping each operation to the appropriate class at the class level and rationalize the interface of each class. To meet more requirements and reach the requirements of $u$ higher reusability, the two phases of object-oriented software continue to be expanded and consolidated. One is to expand to meet the new requirements and consolidate to make the software more general. This cycle is inevitable, but good design is robust to the change of requirements, which can avoid refactoring to some extent. Design pattern records the design structure produced by refactoring, and provides the goal for refactoring.

According to the general characteristics of gridless algorithm, combined with the general problem types of computing software, we can abstract the software framework design into the following basic classes. This paper gives the basic responsibilities and functions of each class, and indicates the recommended design pattern in square brackets.

Cproblem problem class:

A) Determine the type of problem (equation). CFD software generally solves potential flow equation, Euler equation, NS equation, etc. in order to meet the different needs of different users, it is considered to design an extensible equation type 
problem class.

B) To determine the solution space of the problem, the boundary / surface conditions are considered in two-dimensional and three-dimensional space respectively, and the user inputs to determine the boundary / surface conditions.

C) Request / create algorithm object. According to the user's requirements and equation types, various explicit and implicit algorithm objects are created.

D) Request / create flow field objects. According to the solution space, boundary / surface conditions and different flow field discretization methods, the corresponding flow field objects are requested / created.

E) Execute algorithm [facdae]. The task of solving the problem is given to algorithm object and flow field object.

F) The calculation results are output to avoid the calculation deviation caused by the specific traversal order. Under the same experimental conditions, the information interaction between the numerical solution of meshless PDE and that of meshed PDE is compared.

\section{B. Information Interaction Comparison}

In a specific software system, the way of information interaction also affects the scalability and reusability of software. The following is an example of cgird flow field created by Cproblem problem object. Combined with Abstract mode, it shows how to implement scalable design for different equation types (Indian equation, NS equation, etc.) and different flow field discretization methods [15].

In this case, the client can be the function of the CreateGrid of the specific problem object. Through the dynamic binding of Grid Factory port (abstract factory class) and cgridzd (two-dimensional flow field class), the design with good expansibility is realized, and the unnecessary reconstruction caused by the disorder of correlation between the flow field discrete method and space type is avoided. If the flow field is generated step by step and the algorithm is integrated, it is necessary to request an algorithm object in the abstract factory to achieve better function.

Compare the information interaction between the numerical solution of meshless PDE and that of meshed PDE, as shown in Fig. 2:

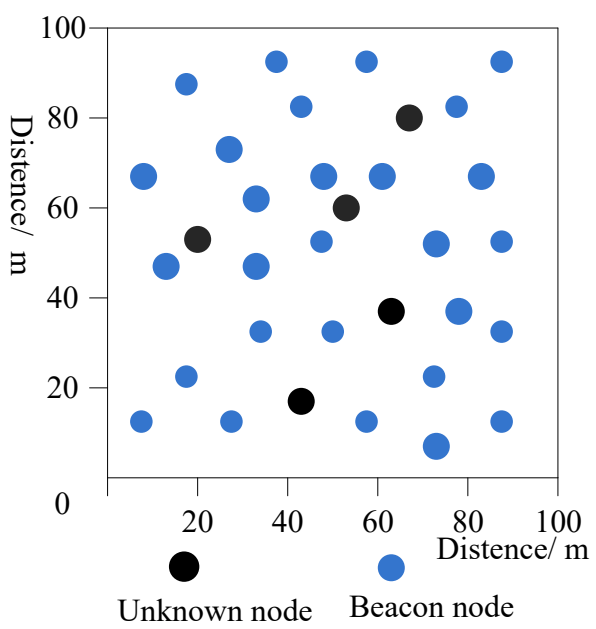

(a) information interaction of numerical solutions for PDEs with grids

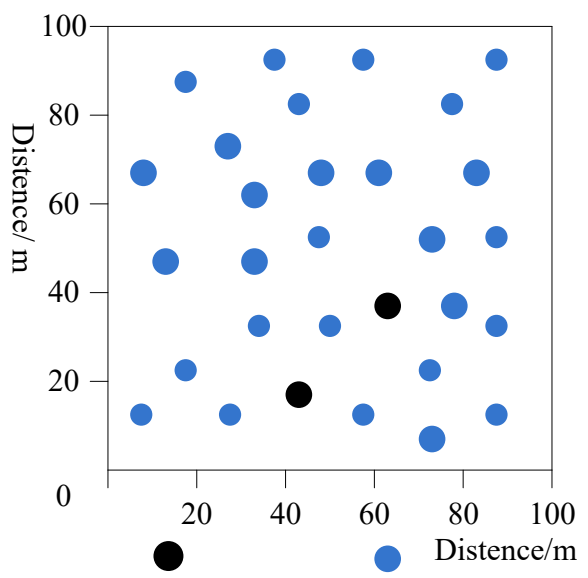

Unknown node Becon code

(b) information interaction of numerical solutions for meshless partial differential equations

Fig. 2 comparison of information interaction between numerical solutions of meshless PDEs and grid PDEs

According to Fig. 2, it can be seen that the interaction of numerical solution information of gridless PDEs is greater than that of gridded PDEs. In the meshless algorithm, the physical quantity calculation of discrete points in the flow field is carried out by requesting the corresponding point cloud instance, and the whole flow field is composed of multiple discrete points, and point cloud and flow field are aggregation objects. In the operation design of aggregate objects, iterator pattern is very necessary. The iterator pattern provides a sequential access to elements of an aggregate object without exposing its internal representation. Its biggest advantage is to separate the ergodic mechanism from the aggregate object, so that we can define different iterators to implement different ergodic strategies, and can switch flexibly in practical application, so as to eliminate some calculation deviation caused by specific ergodic order.

In the actual calculation, the density of discrete points may affect the calculation effect of some or the whole flow field area. Considering the robustness of the software, it is recommended to use the observer mode. This mode defines a one to many 
dependency between objects. When the state of an object (a flow field area) changes (the calculation effect needs to be improved), all objects (point clouds and points belonging to the area) that depend on it are notified and automatically updated (encrypted). The numerical solution of gridless PDE improves the expansibility and reusability of software design on the premise of ensuring software efficiency as much as possible. Of course, the application selection of patterns is not unique and independent, but the best design in practical work requires many design patterns, which fit and interweave to form a larger whole.

\section{Error Comparison of Numerical Results}

In order to compare the approximate accuracy of the meshless PDE function and other functions on the lmaps, the lmaps method based on these two functions is used to solve the PDE in the irregular and regular regions, and the error analysis is given. The KD tree algorithm is selected to search the adjacent nodes in the local area during the solution process, and the approximate accuracy and calculation efficiency are improved through the ripple LOOCV algorithm and the parameter $\mathrm{C}$ is continuously debugged. The maximum absolute error (MAE) and maximum relative error (MRE) are defined as follows:

$$
\begin{aligned}
& M A E=\max \left|u\left(x_{j}, t^{k}\right)-u\left(x_{j}, t^{k}\right)\right| \\
& M R E=\max \left|\frac{u\left(x_{j}, t^{k}\right)-u\left(x_{j}, t^{k}\right)}{u\left(x_{j}, t^{k}\right)}\right|
\end{aligned}
$$

where $\mathrm{k}$ is the time layer, $u\left(x_{j}, t^{k}\right)$ and $u\left(x_{j}, t^{k}\right)$ represent the numerical solution and the exact solution on the $\mathrm{K}$ time layer respectively. In this paper, we choose the Poisson equation, which is commonly used in the fields of electrostatics, mechanical engineering and other physical problems, as well as Burgers equation, which is used to solve shock wave, shallow water wave, traffic flow mechanics and other physical problems, as numerical examples to verify the effectiveness of the above two functions. The two-dimensional position equation considering the distribution of irregular points in the irregular region:

$$
\begin{aligned}
& \Delta u=e^{-x-y},(x, y) \in \Omega \\
& u(x, y)=2 e^{x-y},(x, y) \in \partial \Omega
\end{aligned}
$$

According to the calculation results, we can see that the accuracy of the two functions is satisfactory, and the error in the whole irregular calculation area is basically the same, and both of them are smooth. Compared with the functions in this paper, the error of matern function at the corners of irregular boundary appears slight fluctuation.

Table. III. Error comparison of numerical results of two functions at different points

\begin{tabular}{ccccc}
\hline Check the number & \multicolumn{2}{c}{ The method of this paper } & \multicolumn{2}{c}{ Lmaps matern method } \\
& MAE & MRE & MAE & MRE \\
3200 & $9.56 \mathrm{E}-05$ & $9.39 \mathrm{E}-05$ & $1.55 \mathrm{E}-04$ & $1.62 \mathrm{E}-04$ \\
3600 & $4.36 \mathrm{E}-05$ & $4.55 \mathrm{E}-05$ & $5.26 \mathrm{E}-05$ & $5.17 \mathrm{E}-05$ \\
4000 & $3.69 \mathrm{E}-05$ & $3.70 \mathrm{E}-05$ & $5.73 \mathrm{E}-05$ & $5.90 \mathrm{E}-05$ \\
4400 & $4.65 \mathrm{E}-05$ & $4.60 \mathrm{E}-05$ & $8.35 \mathrm{E}-05$ & $8.08 \mathrm{E}-05$ \\
\hline
\end{tabular}

$\begin{array}{lllll}4800 & 1.37 \mathrm{E}-05 & 8.86 \mathrm{E}-05 & 9.80 \mathrm{E}-05 & 9.78 \mathrm{E}-05\end{array}$

Table III error results of two functions. Generally speaking, the more points, the denser the grid, the higher the accuracy of the numerical solution, but this is not the case. On the contrary, as the number of points increases, the error of matren function also increases. When it increases to a certain value, the error of the function fluctuates greatly and tends to increase. This method is different from the lmaps matern method. With the increase of the number of points, the error shows a small fluctuation trend. When it increases to a certain value, the error is always less than that of the lmaps matern method, which shows that the error is not only related to the total number of nodes, but also closely related to the distribution of nodes. In general, the numerical results obtained by the function in this paper are more accurate and effective than those obtained by the lmaps matern method.

Based on the above, the time taken by different methods to obtain the optimal numerical solution of meshless PDEs is compared. The specific comparison results are shown in Table IV.

Table. IV. Time taken to obtain the optimal numerical solution

\begin{tabular}{ccc}
\hline $\begin{array}{c}\text { Number of } \\
\text { experiments }\end{array}$ & $\begin{array}{c}\text { The method of this } \\
\text { paper }\end{array}$ & $\begin{array}{c}\text { Lmaps matern } \\
\text { method }\end{array}$ \\
\hline 10 & $1.23 \mathrm{~s}$ & $2.58 \mathrm{~s}$ \\
20 & $1.56 \mathrm{~s}$ & $2.69 \mathrm{~s}$ \\
30 & $1.25 \mathrm{~s}$ & $3.55 \mathrm{~s}$ \\
40 & $1.45 \mathrm{~s}$ & $3.24 \mathrm{~s}$ \\
50 & $1.47 \mathrm{~s}$ & $2.54 \mathrm{~s}$ \\
60 & $1.31 \mathrm{~s}$ & $2.84 \mathrm{~s}$ \\
70 & $1.24 \mathrm{~s}$ & $2.96 \mathrm{~s}$ \\
80 & $1.11 \mathrm{~s}$ & $3.14 \mathrm{~s}$ \\
90 & $1.26 \mathrm{~s}$ & $3.47 \mathrm{~s}$ \\
100 & $1.34 \mathrm{~s}$ & $3.55 \mathrm{~s}$ \\
\hline
\end{tabular}

By analyzing the data in Table IV, it can be seen that in 100 experiments, the time used to obtain the optimal numerical solution by this method varies from $1.11 \mathrm{~s}$ to $1.56 \mathrm{~s}$, and the time used to obtain the optimal numerical solution by lmaps matern method varies from $2.54 \mathrm{~s}$ to $3.55 \mathrm{~s}$, which is much higher than the experimental comparison method, which shows that the time used to obtain the optimal numerical solution of meshless PDEs by this method is shorter, The overall operation efficiency is higher and the practical application effect is better, which proves the advantages of this method.

\section{Discussion}

As a numerical method to overcome the shortcomings of the finite element method, the meshless method has attracted the attention of many scholars. Now it has been applied to the related fields of engineering calculation. At the same time, gridless method is still a research field that needs to be developed and improved, and there are many problems that need to be studied in detail. Some problems are discussed and some results are obtained. This paper introduces several 
function approximation methods and PDEs solving methods of meshless method. By comparing the information interaction between the numerical solution of gridless PDEs and that of gridded PDEs, it is proved that the numerical solution of gridless PDEs is superior, and its absolute error and relative error is lower, and the result is more accurate. Compared with the experimental comparison method, this method takes less time to obtain the optimal numerical solution of meshless PDEs, has higher overall operation efficiency and better practical application effect. The reason is that this method uses the meshless method to solve, which overcomes the problems of slow solution speed and low accuracy in the traditional method, and can be further popularized in practice in order to promote the further development of related fields.

\section{CONCLUSION}

This paper mainly studies the basic mechanism of the meshless algorithm, combined with the distance weighting factor, in the process of solving the equation, this paper successfully applies the meshless algorithm to obtain high-precision calculation results. In this paper, the grid free algorithm is used to improve the reusability and flexibility of the software. The basic functional classes of this kind of software are divided, and a series of common design patterns are proposed, which makes a useful exploration for the design of the overall framework of the software. It is true that there are still many problems to be solved in the current meshless computing method, but there is no doubt that this method has a broad application prospect in computational fluid dynamics.

\section{References}

[1] H. Xu, C.D. Cantwell, C, "Monteserin, C. Eskilsson, A.P. Engsig-Karup, and S.J. Sherwin, Spectral/hp element methods: recent developments, applications, and perspective," Journal of Hydrodynamics, vol. 30, no. 1, pp. 1-22, 2018.

[2] K. Hu, X.N. Pang, H.F. Huang, and P. Zhang, "A study on CFD numerical simulation verification about mathematical model of short-circuit high-pressure gas blowing in submarine," Computer Simulation, vol. 36, no. 8, pp. 5-8,14, 2019.

[3] T.T. Zhang, Z.G. Wang, W. Huang, and X.Y. Yan, "Performance comparison between waverider and wide-speed-range gliding vehicle based on CFD approaches," Science China (Technological Sciences), vol. 62, no. 10, pp. 1861-1870, 2019.

[4] M.J. Xiong, J.S. Zhang, W.S. Zhang, and C.T. Yin, "Heterogeneous tide-surge interaction during co-occurrence of tropical and extratropical cyclones in the radial sand ridges of the southern Yellow Sea," Journal of Oceanology and Limnology, vol. 37, no. 6, pp. 1879-1898, 2019.

[5] W.G. Hong, F. Wang, S.N. Wu, and J.C. Xu, "A unified study of continuous and discontinuous Galerkin methods," Science China (Mathematics), vol. 62, no. 1, pp. 1-32, 2019.
[6] S. Irfan, and F. Siddiqui, "A review of recent advancements in finite element formulation for sandwich plates, $\}$ "Chinese Journal of Aeronautics, vol. 32, no. 4, pp. 785-798, 2019.

[7] M.B. Liu, and Z.L. Zhang, "Smoothed particle hydrodynamics (SPH) for modeling fluid-structure interactions," Science China (Physics, Mechanics \& Astronomy), vol. 62, no. 8, pp. 5-42, 2019.

[8] C.D. Huang, W. Liu, and G.W. Yang, "Numerical studies of static aeroelastic effects on grid fin aerodynamic performances," Chinese Journal of Aeronautics, vol. 30, no. 4, pp. 1300-1314, 2017.

[9] G.D. Zong, Y.K. Li, and H.B. Sun, "Composite anti-disturbance resilient control for Markovian jump nonlinear systems with general uncertain transition rate," Science China (Information Sciences), vol. 62, no. 2, pp. 101-118, 2019.

[10]Z.Y. Zhao, P. Yao, X.Y. Wang, J.P. Xu, L. Wang, and J.B. $\mathrm{Yu}$, "Reliable flight performance assessment of multirotor based on interacting multiple model particle filter and health degree," Chinese Journal of Aeronautics, vol. 32, no. 2, pp. 444-453, 2019.

[11]T. Long, Z.N. Liang, and Q.H. Liu, “Advanced technology of high-resolution radar: target detection, tracking, imaging, and recognition," Science China (Information Sciences), vol. 62, no. 4, pp. 5-30, 2019.

[12] J.Z. Qiao, X.F. Li, and J.W. Xu, "A composite disturbance

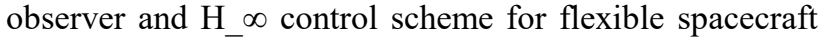
with measurement delay and input delay," Chinese Journal of Aeronautics, vol. 32, no. 6, pp. 1472-1480, 2019.

[13] S.T. Chen, Z.Q. Jian, Y.H. Huang, Y. Chen, Z.L. Zhou, and N.N. Zheng, "Autonomous driving: cognitive construction and situation understanding," Science China (Information Sciences), vol. 62, no. 8, pp. 75-101, 2019.

[14] S.L. Zhang, S. Wang, F.S. Jing, and M. Tan, "Parameter estimation survey for multi-joint robot dynamic calibration case study," Science China (Information Sciences), vol. 62, no. 10, pp. 102-116, 2019.

[15] M. Wang, T.T. Liang, and Z.H. Zhou, "State estimation for non-linear sampled-data descriptor systems: A robust extended kalman filtering approach," Journal of Harbin Institute of Technology (New Series), vol. 26, no. 5, pp. 24-31, 2019.

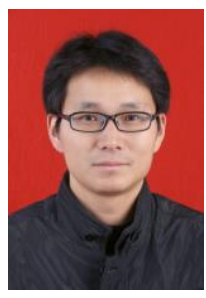

Zhao Zhang, male, was born in December 1982. He holds the 
title of associate professor. He received his bachelor's degree in mathematics and applied mathematics from Anyang Normal University in 2007. He is now working in Zhumadian Vocational and Technical College. His research interests include mathematics education, mathematics and applied mathematics. He has published more than 20 academic papers. At the same time, he also presided over and participated in more than 10 provincial and municipal projects.

\section{Author Contributions:}

Zhao Zhang proposed the numerical solution analysis and comparison of meshlesspartial differential equations (PDEs), and made a specific analysis. It is found that the interaction between the numerical solutions of gridless PDEs is better, and the absolute error and relative error are lower, which proves the superiority of the numerical solutions of gridless PDEs. Creative Commons Attribution License 4.0
(Attribution 4.0 International, CC BY 4.0)

This article is published under the terms of the Creative Commons Attribution License 4.0

https://creativecommons.org/licenses/by/4.0/deed.en_US 